\title{
Laser-Ablative Synthesis of Stable Aqueous Solutions of Elemental Bismuth Nanoparticles for Multimodal Theranostic Applications
}

\author{
Julia C. Bulmahn ${ }^{1}$, Gleb Tikhonowski ${ }^{2}$, Anton A. Popov ${ }^{2}$, Andrey Kuzmin ${ }^{1}{ }^{1}$, \\ Sergey M. Klimentov ${ }^{2}$, Andrei V. Kabashin ${ }^{2,3, * \mathbb{C}}$ and Paras N. Prasad 1,2,* \\ 1 Department of Chemistry and Institute for Lasers, Photonics, and Biophotonics, University at Buffalo, \\ The State University of New York, Buffalo, NY 14260, USA; juliabul@buffalo.edu (J.C.B.); \\ ankuzmin@buffalo.edu (A.K.) \\ 2 Bionanophotonic Lab., Institute of Engineering Physics for Biomedicine (PhysBio), National Nuclear \\ Research University MEPHI, 115409 Moscow, Russia; gtikhonowski@gmail.com (G.T.); \\ aapopov@mephi.ru (A.A.P.); kliment-61@mail.ru (S.M.K.) \\ 3 LP3, Aix Marseille University, CNRS, 13288 Marseille, France \\ * Correspondence: kabashin@lp3.univ-mrs.fr (A.V.K.); pnprasad@buffalo.edu (P.N.P.)
}

Received: 25 June 2020; Accepted: 23 July 2020; Published: 26 July 2020

\begin{abstract}
Elemental bismuth (Bi) nanoparticles (NPs), with the high atomic density of the Bi nuclei, could serve as efficient targeted agents for cancer treatment, with applications such as contrast agents for computed tomography (CT) imaging, sensitizers for image-guided X-ray radiotherapy, and photothermal therapy. However, the synthesis of elemental Bi NPs suitable for biological applications is difficult using conventional chemical routes. Here, we explore the fabrication of ultrapure Bi-based nanomaterials by femtosecond laser ablation from a solid Bi target in ambient liquids and characterize them by a variety of techniques, including TEM, SEM, XRD, FTIR, Raman, and optical spectroscopy. We found that laser-ablative synthesis using an elemental Bi solid target leads to the formation of spherical Bi NPs having the mean size of 20-50 nm and a low size-dispersion. The NPs prepared in water experience a fast (within a few minutes) conversion into 400-500 nm flake-like nanosheets, composed of bismuth subcarbonates, $(\mathrm{BiO})_{2} \mathrm{CO}_{3}$ and $(\mathrm{BiO})_{4} \mathrm{CO}_{3}(\mathrm{OH})_{2}$, while the NPs prepared in acetone demonstrate high elemental stability. We introduce a procedure to obtain a stable aqueous solution of elemental Bi NPs suitable for biological applications, based on the coating of Bi NPs prepared in acetone with Pluronic ${ }^{\circledR} \mathrm{F} 68$ and their subsequent transfer to water. We also show that the laser-synthesized elemental Bi NPs, due to their vanishing band gap, exhibit remarkable absorption in the infrared range, which can be used for the activation of photothermal therapy in the near IR-to-IR window with maximum optical transparency in biological media. Exempt of any toxic synthetic by-products, laser-ablated elemental Bi NPs present a novel appealing nanoplatform for combination image-guided photoradiotherapies.
\end{abstract}

Keywords: bismuth (Bi) nanoparticles; nanosheets; laser ablation in liquids; radiotherapy; phototherapy; nanomedicine

\section{Introduction}

Nanomaterials with high atomic numbers $(Z)$ have demonstrated their ability to act as efficient sensitizers of radiotherapy (RT) [1-3]. When these high-Z elements are irradiated with X-rays, the result is a high local ionization effect leading to DNA strand breaks and ultimately enhancing the efficacy of RT. Off target damage is also limited, as the range of this effect is less than $10 \mathrm{~nm}$; consequently, lower doses of RT are needed to achieve the desired effects [4]. 
From a biomedical prospective, bismuth $(Z=83)$ has several advantages over other high atomic number elements. First, Bi has the highest atomic number of all non-radioactive elements leading to excellent radiosensitization properties. Bi also has a very high X-ray attenuation coefficient $\left(5.74 \mathrm{~cm}^{2} / \mathrm{g}\right.$ at $100 \mathrm{keV}$ ), which leads to its usage in high contrast computed tomography (CT) imaging [5]. Additionally, as a metal, elemental Bi strongly absorbs light over a broad spectral range extending to IR, which allows the resulting photothermal heating of elemental Bi nanoparticles by an IR light to be used for photoacoustic imaging as well as for photothermal therapy, which can synergistically enhance radiotherapy [3]. It is also important to note that Bi demonstrates low toxicity, good biocompatibility, and increased cost effectiveness in comparison to other high-Z elements [2]. Furthermore, Bi can also be easily eliminated from the body due to its favorable reactivity and dissolution properties $[6,7]$. Bi-based compounds have already been successfully explored in CT imaging and radiotherapy [2,3], as well as been used in off-the-shelf medications (e.g., Pepto-Bismol [8]), which confirms their relative safety.

The fabrication of Bi-based nanoparticles (NPs) typically requires chemical pathways, [9-11] but these methods are not fully compatible with stringent requirements of biological systems due to the necessity of using toxic solvents and substances. Based on physical mechanisms of nanostructure formation, laser ablation in liquids is free of limitations of chemical methods, offering a solution to the toxicity problems $[12,13]$. The laser-ablative approach profits from a natural production of nanoclusters under the interaction of pulsed laser radiation with a solid target, which are then released to an ambient liquid to form a colloidal nanoparticle solution [14-16]. When ablated in a pure ambient (deionized water, ethanol, acetone, etc.), the formed NPs are exempt of any toxic contamination, which opens up avenues for the synthesis of different nanomaterials for biological use, including Au NPs [15-18], Si NPs [19,20], TiN NPs [21], and Sm [22]. Methods of laser ablation have already been explored for the fabrication of Bi-based nanomaterials [23-27]. However, laser ablation in water has yielded only stable Bi compound NPs (oxides, carbonates, etc.), which cannot provide the maximum local concentration of $\mathrm{Bi}$ nuclei in comparison to elemental $\mathrm{Bi}$, as a high concentration of $\mathrm{Bi}$ would reduce the needed dose level for radiotherapy and would also enhance the contrast for imaging. Furthermore, the transfer of elemental Bi NPs prepared in organic solutions when transferred to water typically suffers from a prompt oxidation of NPs and destabilization of solutions. These oxidized Bi compounds, having a wide bandgap, do not significantly absorb to effect sufficient photothermal therapy using IR light.

Here, we investigate conditions of synthesis and properties of Bi-based NPs by using methods of femtosecond laser ablation. We report the fabrication of stable aqueous solutions of ultrapure elemental Bi NPs by laser ablation in acetone, followed by their coating with Pluronic ${ }^{\circledR}$ F68 and transfer to water in unchanged form. This suggests that a proper surface modification of laser-synthesized Bi NPs is a plausible method to prepare stable, biocompatible, and pure Bi NPs for applications in nanomedicine.

\section{Materials and Methods}

\subsection{Synthesis of Nanoparticles}

NPs were synthesized by ultrashot (fs) laser ablation of the bismuth (Bi) target (GoodFellow, Coraopolis, PA, USA, purity $99.999 \%)$ in deionized water $\left(18.2 \mathrm{M} \Omega \mathrm{cm}\right.$ at $\left.25^{\circ} \mathrm{C}\right)$ or technical grade acetone, under ambient conditions. A schematic of the experimental setup is shown in Figure 1. The Bi target was fixed vertically on the wall of a quartz vessel filled with $40 \mathrm{~mL}$ of a liquid. A 3-mm-diameter beam from a Yb:KGW laser (1030 nm wavelength, $270 \mathrm{fs}$ pulse duration, $30 \mu \mathrm{J}$ pulse energy, $100 \mathrm{kHz}$ repetition rate; TETA 10 model, Avesta, Moscow, Russia) was focused by a 100-mm F-theta lens on the surface of the target, through a side wall of the ablation vessel. The thickness of the liquid layer along the laser beam was $6 \mathrm{~mm}$. The focusing conditions were set to obtain maximum productivity from the ablation process (defined as ablated mass per duration of ablation) individually for each solvent. The laser beam was moved over a $20 \times 20 \mathrm{~mm}$ area on the surface of the target, with $4000 \mathrm{~mm} / \mathrm{s} \mathrm{speed}$ using a galvanometric scanner. This was done to avoid drilling of a hole in the target and to maximize 
the nanoparticle (NP) yield. The duration of each laser ablation experiment was 15 min. The target and the ablation chamber were cleaned after each ablation experiment, by using an ultrasonication step in acetone, followed by ultrasonication in water, thorough rinsing in ultrapure water and, finally, drying under ambient conditions.

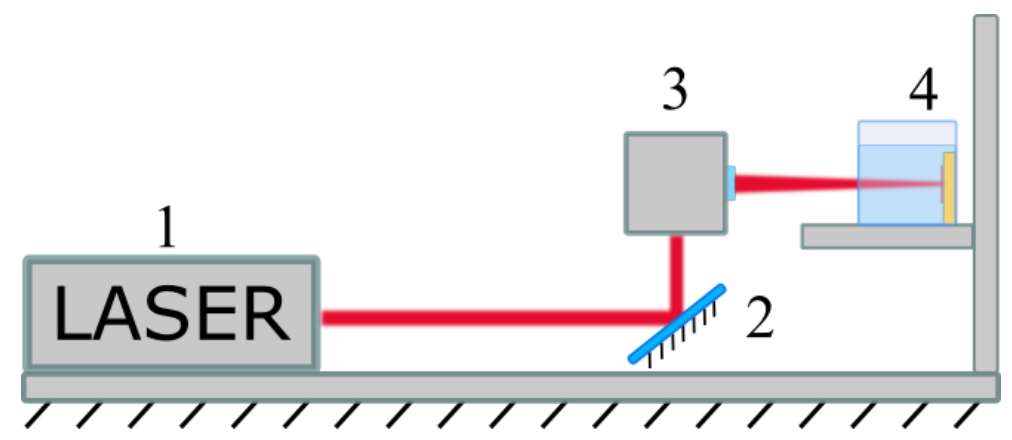

Figure 1. Schematic representation of the experimental setup for laser ablation. 1. a laser unit; 2. a mirror; 3. a galvanometric scanner and a focusing F-theta lens; 4 . The ablation chamber with the Bi target submerged in liquid.

\subsection{Surface Modification of Bi NPS}

Bi NPs ablated in acetone were successfully transferred to water using a surface coating of Pluronic ${ }^{\circledR}$ F68 (Sigma-Aldrich, St. Louis, MO, USA). To achieve this surface coating, $50 \mathrm{mg}$ of Pluronic (R) F68 was added to $10 \mathrm{mg}$ of Bi NPs in acetone, and this was vortexed for $30 \mathrm{~s}$ to dissolve the polymer. This solution was then evaporated to dry using moderate air flow and resuspended in $1 \mathrm{~mL}$ distilled water with sonication. The resulting dispersion was centrifuged $10 \mathrm{~min}$ at $3000 \mathrm{RCF}$ and the supernatant was discarded to remove any excess polymer. The resulting pellet was resuspended in $1 \mathrm{~mL}$ deionized water to produce a stable brown dispersion. The NPs were stored at RT for further use.

\subsection{Characterization of Nanoparticles}

Morphology, structure, size and composition of NPs and nanosheets were characterized by transmission electron microscopy (TEM) using a JEM-2010 microscope (JEOL USA, Inc., Peabody, MA, USA) at an acceleration voltage of $200 \mathrm{kV}$ or scanning electron microscopy (SEM) using a MAIA 3 microscope (Tescan, Czech Republic) operating at $0.1-30 \mathrm{kV}$ accelerating voltage. Samples for electron microscopy were prepared by dropping $10 \mu \mathrm{L}$ of the NPs solution onto a formvar-coated copper grid (for TEM) or cleaned crystalline silicon substrate (for SEM), with subsequent drying at ambient conditions. The hydrodynamic diameter and $\zeta$-potential measurements were performed using a 90Plus zeta sizer (Brookhaven Instruments, Holtsville, NY, USA). The powder X-ray diffraction (XRD) patterns were recorded by a Rigaku Ultima IV X-Ray Diffractometer (Rigaku, Tokyo, Japan), using $\mathrm{Cu} \mathrm{K} \alpha$ radiation $(\lambda=0.15418 \mathrm{~nm})$. The $2 \theta$ angle of the XRD patterns was recorded at a scanning rate of $2 \% \mathrm{~min}$. The Fourier-transform infrared (FTIR) spectra were recorded using a Spectrum II FTIR spectrophotometer with a micro-Attenuated Total Reflectance sampling accessory (Perkin Elmer, Waltham, MA, USA). Samples were placed on a 2-mm diamondwindow and spectra were recorded in the wavenumber range of $400-4000 \mathrm{~cm}^{-1}$ with a resolution of $4 \mathrm{~cm}^{-1}$. The force gauge reading was 50 units. ATR correction was applied. Raman spectra were measured using a DXR2 Raman microscopy system (Thermo Fisher Scientific, Madison, WI) with a red laser source (ROUSB-633-PLR-70-1, Ondax, CA, USA) and a Plan $\mathrm{N}$ oil immersion 100x ( $\mathrm{Na}=1.25)$ objective lens (Olympus, Japan). The following measurement parameters were used: $7 \mathrm{~mW}$ Continuous Wave power on the sample, $1 \mathrm{~s}$ of accumulation time, and $50 \mu \mathrm{m}$ of confocal pinhole diameter. Raman spectra were collected and processed by the OMNIC software for dispersive Raman (Thermo Fisher Scientific, Madison, WI, USA). Water dispersed NPs were sealed between a microscope slide and a cover slip, while acetone-dispersed NPs were sealed in a capillary tube $(\varnothing 300 \mu \mathrm{m}, 10 \mathrm{~mm})$. 


\subsection{Photothermal Gradient Measurement}

The temperature distribution dynamics induced by the nanomaterials under $808 \mathrm{~nm}$ laser irradiation was monitored by a thermal imaging camera FLIR A600 (FLIR Systems, Wilsonville, OR, USA). For thermal gradient dynamics measurements, all samples were prepared at a concentration of $100 \mu \mathrm{g} / \mathrm{mL}$ and transferred into capillary tubes (ø $300 \mu \mathrm{m}, 10 \mathrm{~mm}$ ) to avoid significant thermal convection of solvent during excitation. Each sample was then imaged in real time upon $808 \mathrm{~nm}$ laser irradiation of the laser beam focused in a $\sim 15 \times 50 \mu \mathrm{m}$ waist inside the tube. Saved sequences of thermal images were processed by a FLIR camera software to plot the change in maximum temperature of the sample over time.

\section{Results and Discussion}

\subsection{Physical Characterization}

Laser ablation of the Bi target in technical acetone (LAA) and deionized water (LAW) resulted initially in dark-brown colloidal solutions (Figure S1). During the first 5-10 min of the ablation process, there was no visible difference in color of solutions being prepared in any solvents; however, by the end of an ablation experiment (15 min), water-based colloidal solution became substantially turbid, while those prepared in acetone remained clear. NPs prepared by LAW further changed their color to milky-white after approximately $100 \mathrm{~min}$. The final appearances of the colloids synthesized are shown in Figure 2. Transmission electron microscopy (TEM) images taken several days after the preparation revealed significant differences in sizes and morphologies of Bi-based nanomaterials synthesized by LAA and LAW (Figure 3). Bi fs LAA results in spherical NPs with sizes from 5 to $50 \mathrm{~nm}$ with an average diameter of $28 \pm 4 \mathrm{~nm}$, while large flake-like nanosheets ranging from 185 to $780 \mathrm{~nm}$ with an average width of $455 \pm 50 \mathrm{~nm}$ and a thickness of 10-20 nm are obtained after Bi fs LAW.

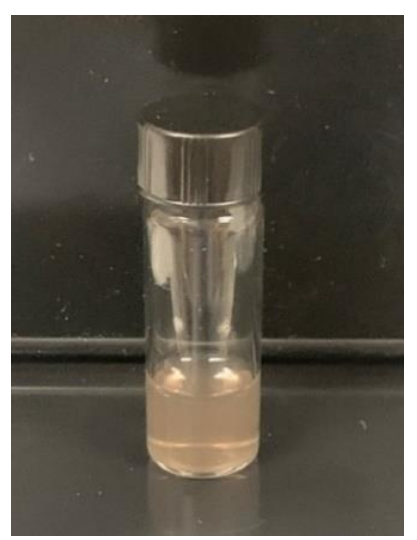

(a)

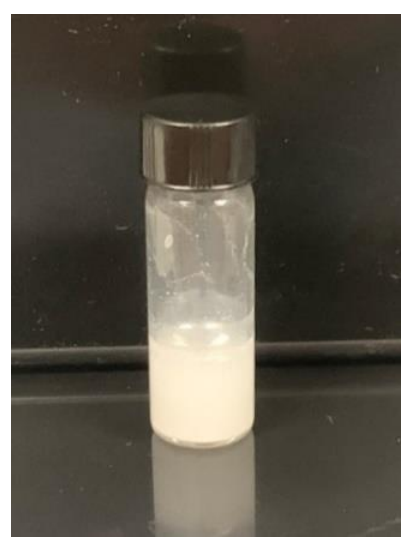

(b)

Figure 2. Colloidal solution of (a) Bi nanoparticles (NPs) produced from fs LAA and (b) Bi nanosheets after fs LAW. 


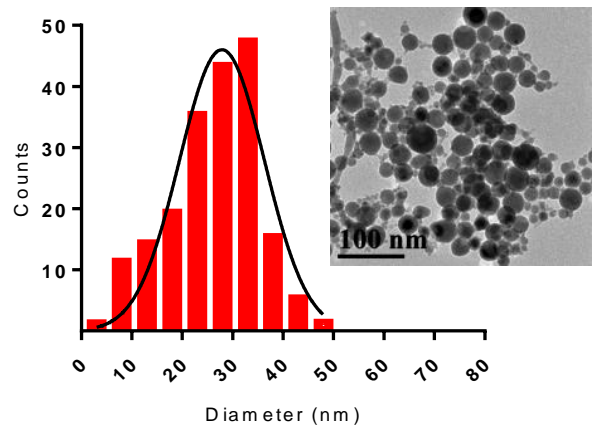

(a)

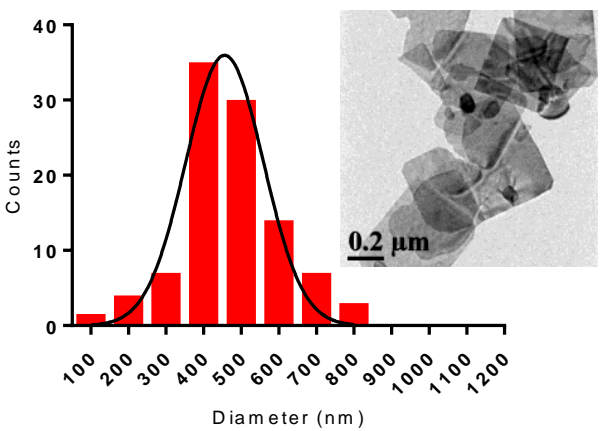

(b)

Figure 3. Size distributions and transmission electron microscopy (TEM) images of (a) Bi NPs produced from fs LAA and (b) Bi nanosheets after fs LAW.

We investigated possible reasons for such profound differences in morphology of the nanomaterials prepared by fs laser ablation in different solvents. Scanning electron microscopy (SEM) images of Bi LAW, performed immediately after the synthesis and several days after, demonstrated that the synthesized nanosheets seemed to grow over time and develop a more crystalline shape (Figure S2). No spherical NPs were observed in the SEM images of the Bi sample after LAW; however, relatively long sample preparation time for electron imaging could be a reason for the absence of observed spherical NPs in the sample. To exclude this possibility, we quickly changed the solvent after Bi LAW from deionized water to acetone by centrifugation ( $1 \mathrm{~min}, 10,000 \mathrm{RCF})$. In this case, we observed spherical NPs with a narrow size distribution and a mean diameter of $50 \mathrm{~nm}$ (Figure 4). We noticed that the presence of water played a decisive role in the final morphology of synthesized nanomaterials; even the addition of $10 \mu \mathrm{L}$ of deionized water to $1 \mathrm{~mL}$ of sample Bi NPs prepared by LAA resulted in a change morphology from spherical NPs to flake-like nanosheets.

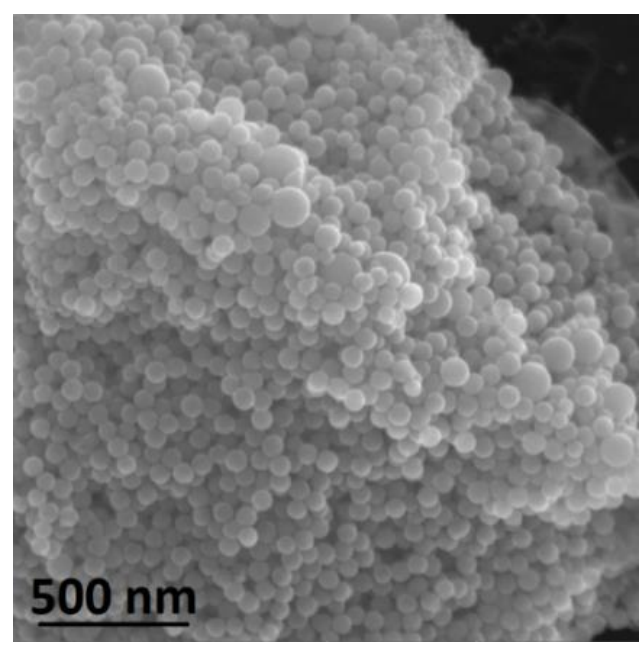

Figure 4. SEM image of Bi NPs produced from fs LAW and re-dispersed in acetone immediately after the synthesis.

Good colloidal stability of laser-synthesized nanomaterials is dictated by the electrical charging of the material during the ablation process and the related electrostatic stabilization. According to our $\zeta$ - potential measurements, the surface potential of Bi NPs prepared by fs LAA was $-20 \mathrm{meV}$, which coincides with the stability threshold for colloidal solutions and is consistent with the observed stability of our sample over time. Not surprisingly, the nanosheets produced as result of Bi fs LAW 
precipitated out over time; despite their $\zeta$ - potential of $-40 \mathrm{meV}$, their large size results in poor colloidal stability [28].

The X-ray diffraction (XRD) pattern of the Bi nanoparticles generated by fs LAA can be seen in Figure 5a. All the peaks can be indexed with the reference data for Bi metal (ICDD, No. 00-44-1246) with lattice parameters, $\mathrm{a}=4.547 \AA$ and $\mathrm{c}=11.862 \AA$; no impurity phase is present, indicating LAA generates pure bismuth metal nanoparticles. The XRD pattern of the nanosheets generated by Bi fs LAW can be seen in Figure $5 \mathrm{~b}$. Most of the diffraction lines are indexed with the reference data for $(\mathrm{BiO})_{2} \mathrm{CO}_{3}$ (ICDD No. 00-025-1464). Some peaks characteristic of $(\mathrm{BiO})_{4} \mathrm{CO}_{3}(\mathrm{OH})_{2}$ (ICDD 00-038-0579) were also observed at $11.99,29.45,36.50$, and $50.19^{\circ}$. This suggests that Bi LAW results in nanosheets containing a combination of both $(\mathrm{BiO})_{2} \mathrm{CO}_{3}$ and $(\mathrm{BiO})_{4} \mathrm{CO}_{3}(\mathrm{OH}) 2[29]$.
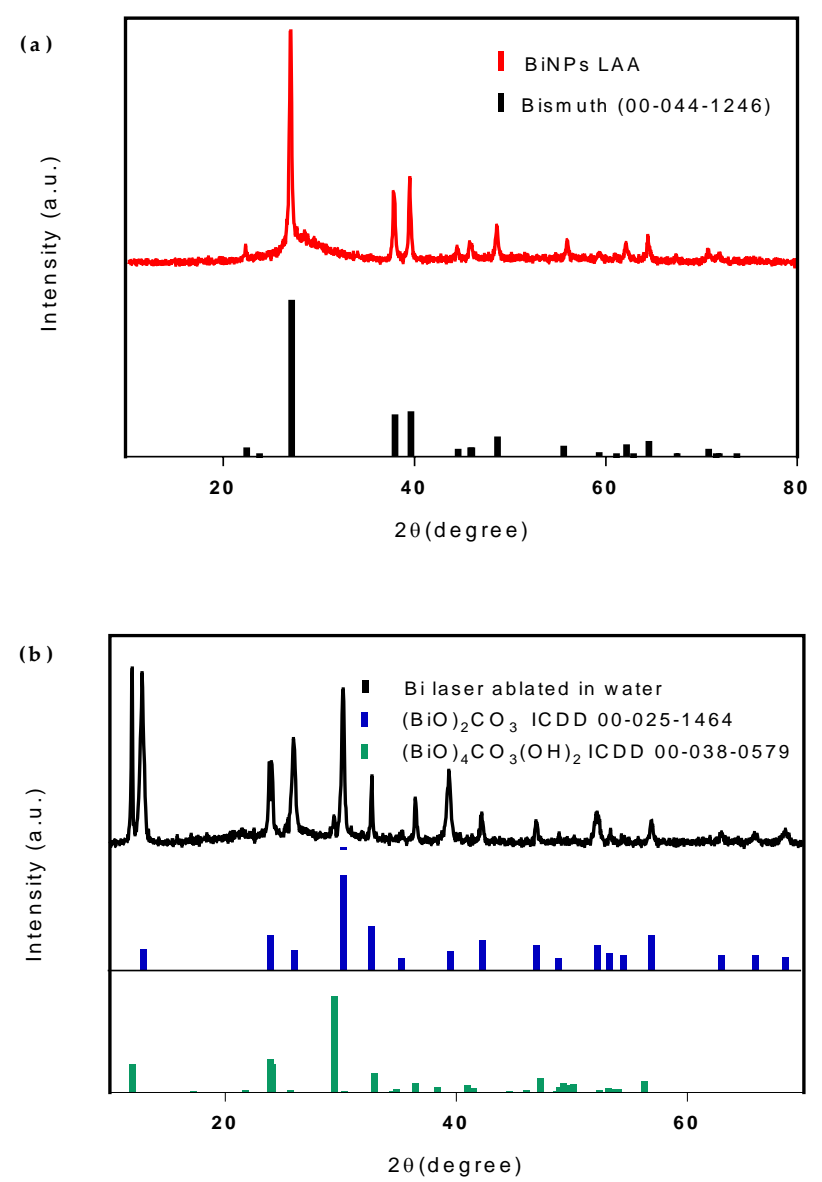

Figure 5. X-ray diffraction (XRD) patterns of (a) Bi NPs produced from fs LAA and (b) Bi nanosheets after fs LAW.

The FTIR spectrum of Bi fs LAA can be seen in Figure 6a. No characteristic peaks from the Bi NPS are detected, most likely due to their infrared inactivity. The FTIR spectrum of Bi fs LAW can be seen in Figure $6 \mathrm{~b}$. The $\mathrm{CO}_{3}{ }^{2-}$ ion, with a point group symmetry of $D_{3 \mathrm{~h}}$, has four internal vibrations, which are all observed in this spectrum. The symmetric stretching mode $\left(v_{1}\right)$ is attributed to the medium weak absorption band observed at $1072 \mathrm{~cm}^{-1}$. The strong absorption band at $1385 \mathrm{~cm}^{-1}$ and the shoulder seen at $1458 \mathrm{~cm}^{-1}$ can be assigned to the anti-symmetric stretching mode $\left(v_{3}\right)$ of the $\mathrm{CO}_{3}{ }^{2-}$ group. The band observed at $848 \mathrm{~cm}^{-1}$ is attributed to the out-of-plane bending mode $\left(v_{2}\right)$, while the bands at 696 and $672 \mathrm{~cm}^{-1}$ can be attributed to the in-plane deformation mode $\left(v_{4}\right)$ of the coordinated $\mathrm{CO}_{3}{ }^{2-}$ group [30]. The strong absorption band that appears at $545 \mathrm{~cm}^{-1}$ is representative of the stretching modes of the $\mathrm{Bi}-\mathrm{O}$ bonds in $(\mathrm{BiO})_{2} \mathrm{CO}_{3}$. This supports the hypothesis that $\mathrm{Bi} \mathrm{LAW}$ produces $(\mathrm{BiO})_{2} \mathrm{CO}_{3}$ 
nanosheets. The weakly observed $\mathrm{O}-\mathrm{H}$ stretch at $3475 \mathrm{~cm}^{-1}$ also suggests the possible presence of $(\mathrm{BiO})_{4} \mathrm{CO}_{3}(\mathrm{OH})_{2}$ or uncoordinated water left over after drying [31,32]
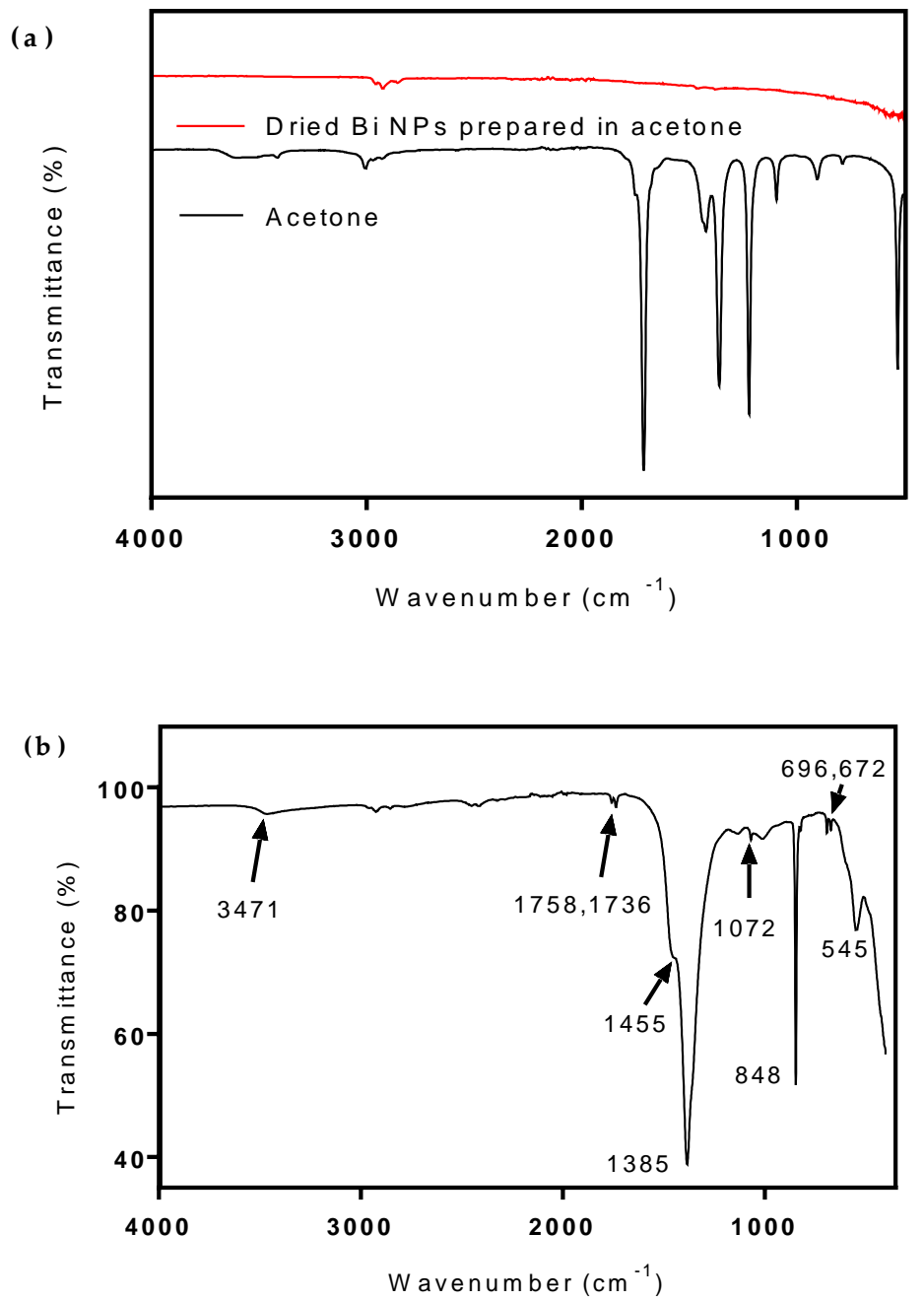

Figure 6. Fourier-transform infrared (FTIR) spectra of (a) Bi NPs produced from fs LAA and (b) Bi nanosheets after fs LAW.

The Raman spectrum of Bi fs LAA can be seen in Figure 7a. The Raman bands located at 68 and $94 \mathrm{~cm}^{-1}$ can be assigned to first order $E_{g}$ and $A_{1 g}$ phonon modes, respectively, while a weak second order band is visible at $184 \mathrm{~cm}^{-1}$. The bands at 126 and $313 \mathrm{~cm}^{-1}$ correspond to unique $\mathrm{Bi}-\mathrm{O}$ stretches, which are attributed to the $\beta$-phase $\mathrm{Bi}_{2} \mathrm{O}_{3}$ [33]. $\mathrm{Bi}_{2} \mathrm{O}_{3}$ impurities were observed in the Raman spectrum but not in the FTIR spectrum or the XRD pattern. This is consistent with laser irradiation-induced oxidation of the bismuth surface, as previously demonstrated by Lewis et al. They demonstrated that for sufficiently large power densities an oxidation reaction occurs, followed by a rearrangement into $\beta-\mathrm{Bi}_{2} \mathrm{O}_{3}$ [34]. The Raman spectra of $\mathrm{Bi}$ fs $\mathrm{LAW}$ can be seen in Figure $7 \mathrm{~b}, \mathrm{c}$. The Raman bands below $600 \mathrm{~cm}^{-1}$ are consistent with the lattice and $\mathrm{Bi}-\mathrm{O}$ vibrations previously reported for $(\mathrm{BiO})_{2} \mathrm{CO}_{3}$ and $(\mathrm{BiO})_{4} \mathrm{CO}_{3}(\mathrm{OH})_{2}$ [35]. The two bands observed in this spectrum, 244 and $275 \mathrm{~cm}^{-1}$, are not seen in the Raman spectrum of pure $(\mathrm{BiO})_{2} \mathrm{CO}_{3}$ and have been previously been assigned to the concerted motions of the coordinated $\mathrm{OH}$ groups [35]. The strong band at $1067 \mathrm{~cm}^{-1}$ is representative of the $v_{1}$ mode of the coordinated $\mathrm{CO}_{3}{ }^{2-}$ group, while the bands at 1360 and $666 \mathrm{~cm}^{-1}$ are attributed to the $v_{4}$ and $v_{3}$ modes, respectively. The $v_{2}$ mode is not Raman active and therefore not observed [35]. These spectra support the conclusion that $\mathrm{Bi}$ fs LAW results in a combination of $(\mathrm{BiO})_{2} \mathrm{CO}_{3}$ and $(\mathrm{BiO})_{4} \mathrm{CO}_{3}(\mathrm{OH})_{2}$. 

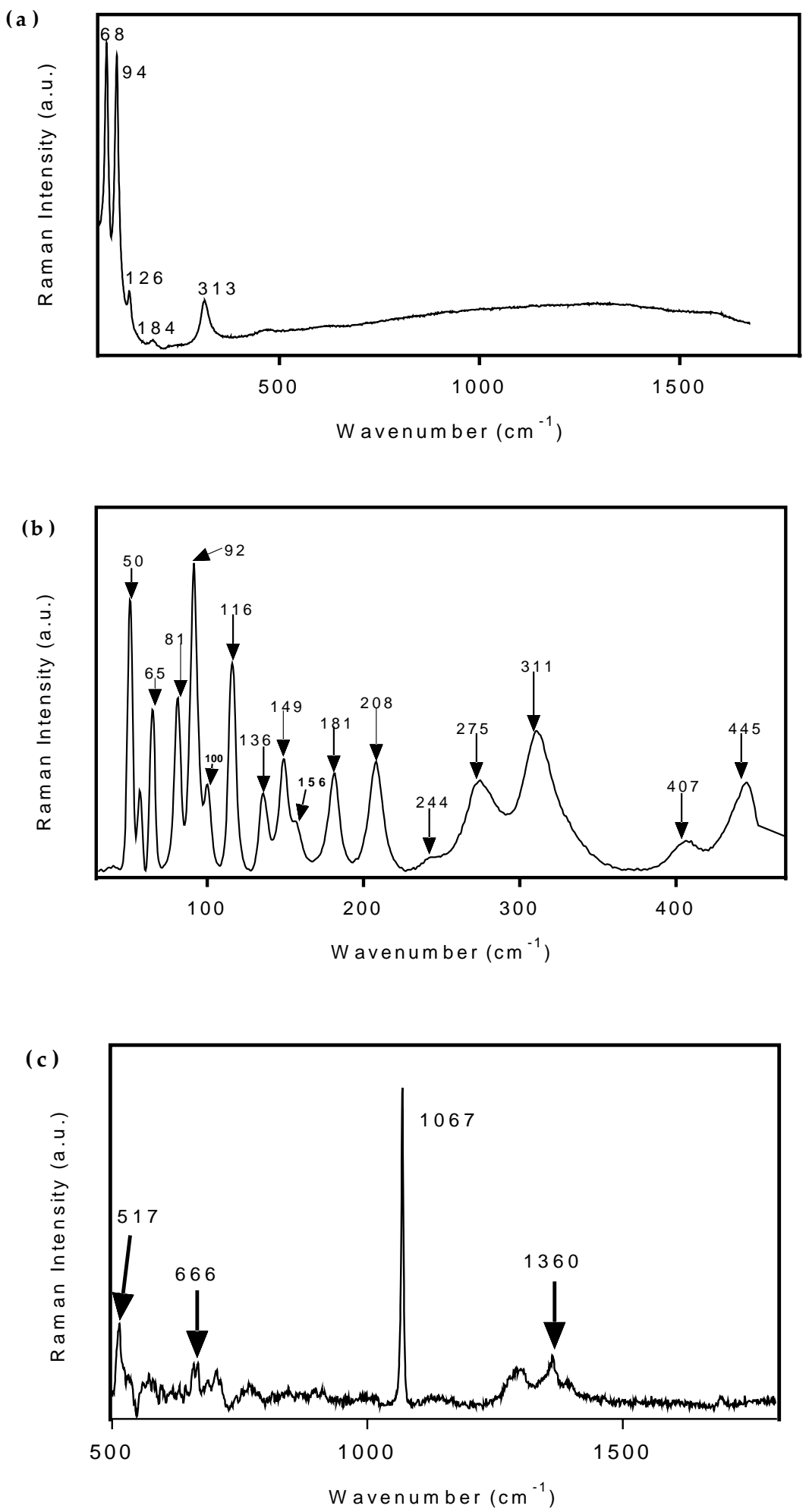

Figure 7. Raman spectra of (a) Bi NPs produced from fs LAA and (b,c) Bi nanosheets after fs LAW.

UV/VIS/Nearlinfrared(NIR)spectroscopy was used to characterize the optical absorption properties of the materials. The absorbance spectra of Bi LAA and Bi LAW can be seen in Figure 8; it is featureless and continuous above $400 \mathrm{~nm}$. This spectrum is representative of previously reported pure elemental Bi NPs; however, they typically have an absorbance peak below $300 \mathrm{~nm}[36,37]$. This peak cannot be observed in the Bi LAA sample due to the high absorbance of acetone below $300 \mathrm{~nm}$ [27]. The absorbance 
of Bi LAW lies mainly below $400 \mathrm{~nm}$ in the ultraviolet region, with a maximum absorbance between 230 and $280 \mathrm{~nm}$; this is consistent with that seen for $(\mathrm{BiO})_{2} \mathrm{CO}_{3}[38,39]$.

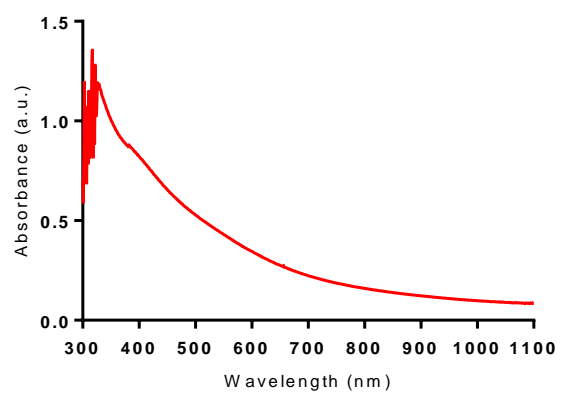

(a)

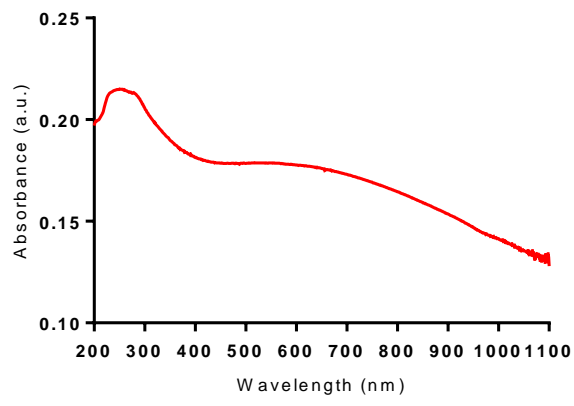

(b)

Figure 8. VIS-NIR absorbance spectrum of (a) Bi NPs produced from fs LAA and UV-VIS-NIR absorbance spectrum of (b) Bi nanosheets after fs LAW.

To determine the potential of the synthesized Bi NPs and nanosheets to be used for localized heating under NIR (808 nm) excitation, we studied the dynamics of thermal heating for both nanomaterials. The results of this study (Figure 9) show that elemental Bi NPs, but not Bi nanosheets, demonstrated a significant increase in temperature after $30 \mathrm{~s}$ exposure to the NIR excitation. The initial rise in temperature of the elemental Bi NPs upon exposure is approximately $2{ }^{\circ} \mathrm{C} /$ second for the first $5 \mathrm{~s}$ reaching a temperature of $31.5^{\circ} \mathrm{C}$. This rate levels off to approximately $0.4^{\circ} \mathrm{C} /$ second over the remaining $25 \mathrm{~s}$, leading to a temperature maximum of $39^{\circ} \mathrm{C}$, for an overall increase of $16.5^{\circ} \mathrm{C}$. The Bi nanosheets only demonstrate a $4.5^{\circ} \mathrm{C}$ increase in temperature over the same $30 \mathrm{~s}$ exposure period. This result shows the relative merit of elemental Bi NPs for near IR photothermal therapy and as a contrast agent for near IR photoacoustic imaging.

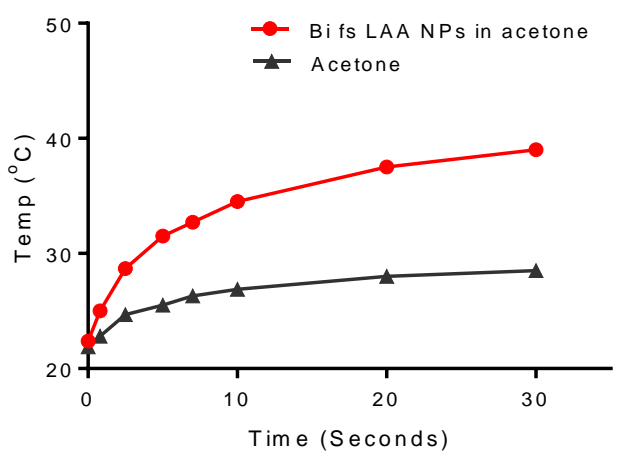

(a)

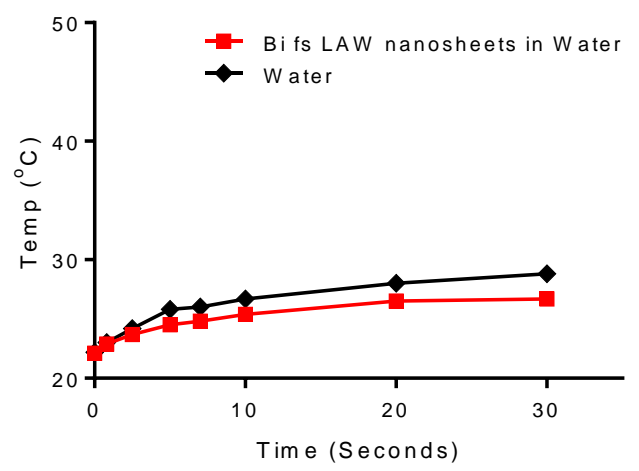

(b)

Figure 9. Photothermal heating of (a) Bi NPs produced from fs LAA and (b) Bi nanosheets after fs LAW, using an 800-nm excitation source at 1-watt power.

\subsection{Stability Studies}

To create a stable dispersion of Bi NPs in water, Pluronic ${ }^{\circledR}$ F68 was used to coat the surface and prevent the conversion of the NPs from pure Bismuth metal to oxide or carbonate compounds. The stability of the Bi NPs in water was evaluated by observing changes in color, hydrodynamic diameter, and Raman spectra of coated elemental Bi NP water dispersion over 14 days. Figure 10 demonstrates the color stability of the Bi NPs dispersion over time. The uncoated Bi NP dispersion in water changes from brown to white after 1 day (Figure S3), while the coated Bi NP dispersion maintains its brown color for more than 14 days. Additionally, aside from a slight increase in the hydrodynamic diameter due to the presence of the polymer surface coating, the coated Bi NPs maintain their size for 
more than 14 days, while a dramatic increase in the hydrodynamic diameter, from $\sim 50$ to $\sim 400 \mathrm{~nm}$, is observed in the uncoated Bi NPs in water after just 1 day (Figure 10 and Figure S3). This suggests that the coated Bi NPs are not converting to oxide or carbonate compounds, as is occurring with the uncoated Bi NPs.
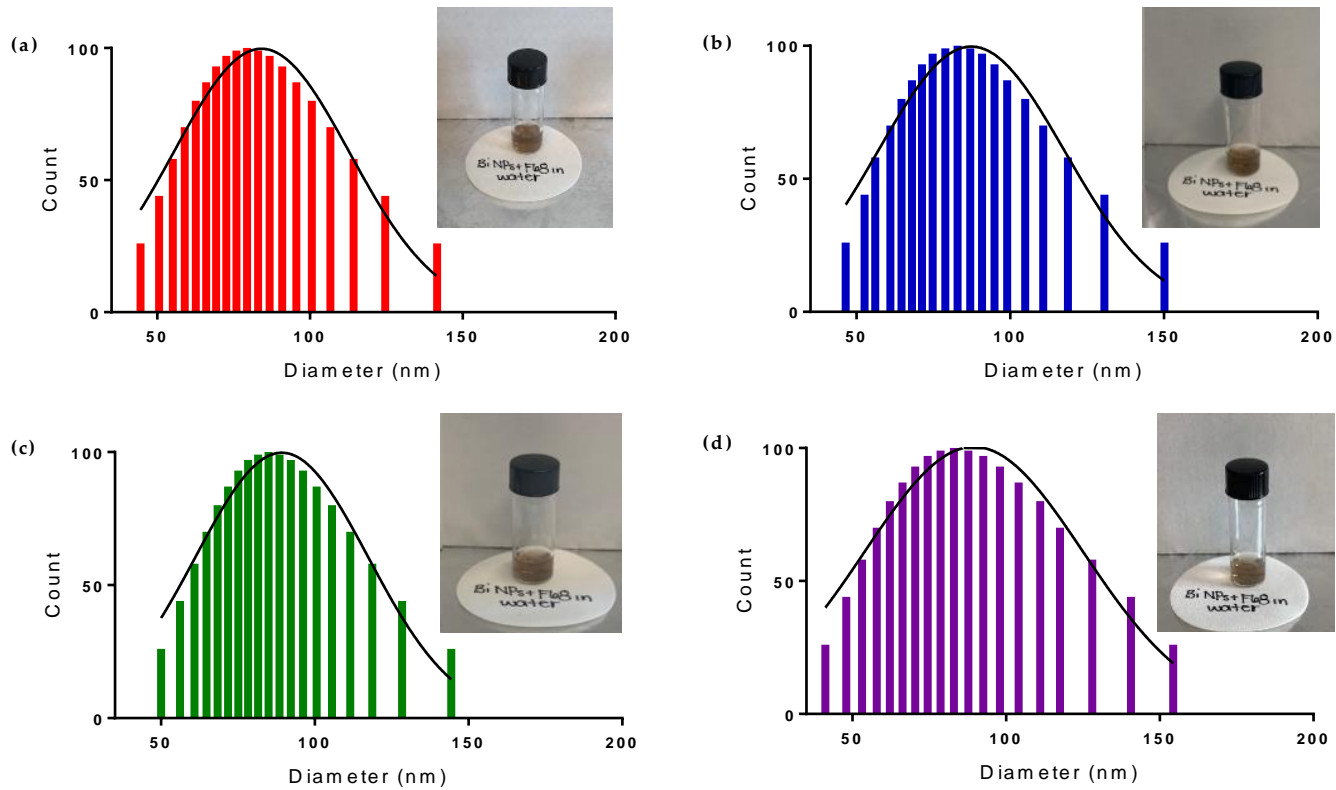

Figure 10. Colloidal solutions and size distributions, determined by Dynamic Light Scattering (DLS), of pluronic ${ }^{\circledR}$ F68 coated Bi NPs (a) immediately after, (b) 5 days after, (c) 10 days after, and (d) 14 days after transfer to water.

To confirm that the retention of color and size are indicative of the stability of the coated Bi NPs in water, we simultaneously evaluated the chemical composition of the NPs using Raman spectroscopy (Figure 11). The strong Raman bands located at 68 and $94 \mathrm{~cm}^{-1}$ in all four samples confirm the retention of pure Bi metal NPs after transfer to water using Pluronic ${ }^{\circledR}$ F68. The absence of the strong band at $1067 \mathrm{~cm}^{-1}$, representative of the $v_{1}$ mode of $\mathrm{CO}_{3}{ }^{2-}$, confirms that the sample of coated Bi NPs in water contains no $\mathrm{CO}_{3}{ }^{2}$. Additionally, the absence of the bands at 126 and $313 \mathrm{~cm}^{-1}$, which correspond to the unique $\mathrm{Bi}-\mathrm{O}$ stretches of $\beta$-phase $\mathrm{Bi}_{2} \mathrm{O}_{3}$, confirms that we have successfully prepared stable elemental $\mathrm{Bi}$ NPs in water using this coating method. This is also supported by the XRD pattern of the coated NPs (Figure S4), which can be indexed with the reference data for Bi metal (ICDD, No. 00-44-1246) without the presence of any impurity phase. This reinforces the conclusion that the F68 coated Bi NPs are not undergoing the conversion to $(\mathrm{BiO})_{2} \mathrm{CO}_{3}$ when transferred to water.
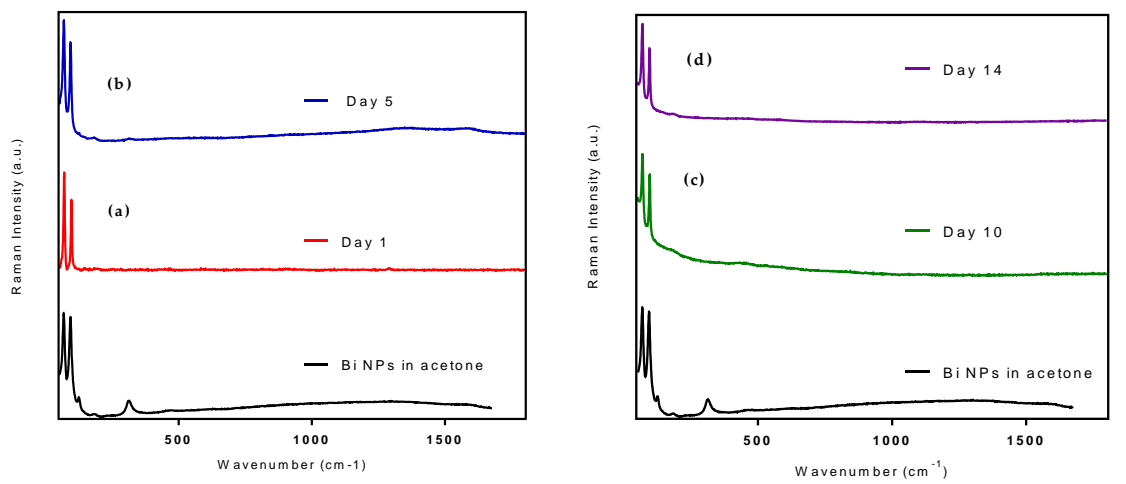

Figure 11. Raman spectra of Pluronic ${ }^{\circledR}$ F68 coated Bi NPs (a) immediately after, (b) 5 days after, (c) 10 days after, and (d) 14 days after transfer to water, compared with as-synthesized Bi NPs in acetone. 
Lastly, we wanted to evaluate the dynamics of thermal heating for the Pluronic ${ }^{\circledR}$ F68 coated Bi NPs in water to confirm that this transfer does not reduce their localized heating potential. The results of this study (Figure 12) show that the coated Bi NPs demonstrated a significant increase in temperature, greater than that of the NPS in acetone, after $30 \mathrm{~s}$ exposure to the NIR excitation. Upon exposure, the initial rise in temperature of the coated Bi NPs in water is approximately $2{ }^{\circ} \mathrm{C} / \mathrm{s}$ for the first $5 \mathrm{~s}$ reaching a temperature of $37^{\circ} \mathrm{C}$. This increase rate slows down to $1.0^{\circ} \mathrm{C} / \mathrm{s}$ over the next $5 \mathrm{~s}$, and levels off at $0.5{ }^{\circ} \mathrm{C} /$ second for the remaining $20 \mathrm{~s}$ to finally yield a temperature maximum of $53{ }^{\circ} \mathrm{C}$, for an overall increase of $24.4^{\circ} \mathrm{C}$. This suggests that the coated NPs transferred to water will be effective for use in photothermal therapy in vivo as well as for photoacoustic imaging.

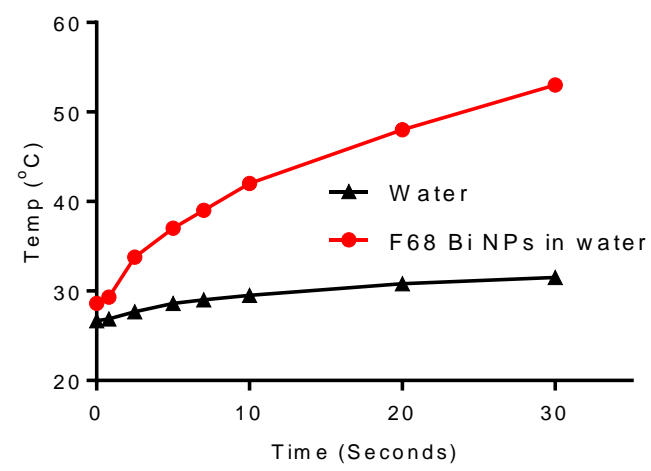

Figure 12. Photothermal heating of Pluronic ${ }^{\circledR}$ F68 coated Bi NPs, 10 days after transfer to water, using an 800-nm excitation source at 1-watt power.

\section{Conclusions}

We elaborated methods of femtosecond laser ablation from a Bi target in liquid ambient to fabricate Bi-based nanomaterials for biomedical applications. We showed that Bi-based NPs prepared in deionized water rapidly convert into 400-500 nm flake-like nanosheets composed of bismuth subcarbonates, while the NPs prepared in acetone present stable solutions of crystalline elemental Bi NPs having the mean size of 20-40 nm and a low size-dispersion. We also showed that Bi NPs prepared in acetone can be transferred to water via coating with Pluronic ${ }^{\circledR}$ F68. After such a transfer, aqueous NPs solutions demonstrated a high stability (stability characteristics were recorded for at least two weeks) and are very suitable for use in biological systems. It was also found that the NPs could exhibit remarkable absorption in the infrared range, making them a promising nanoplatform for photothermal therapy. Based on obtained physico-chemical characteristics and exceptional purity, laser-synthesized NPs promise a major advancement of current methods for photo/radiotherapies.

Supplementary Materials: The following are available online at http:/www.mdpi.com/2079-4991/10/8/1463/ s1, Figure S1. Colloidal solutions of Bi-based nanostructures immediately after (a) fs LAW and (b) fs LAA, Figure S2. SEM images of Bi nanosheets prepared by LAW (a) immediately after synthesis and (b) several days after, Figure S3. Colloidal solutions and size distributions, determined by DLS, of uncoated Bi NPs (a) immediately after and (b) 1 day after transfer to water, Figure S4. X-Ray Diffraction (XRD) patterns of Pluronic®F68 coated Bi NPs 10 days after transfer to water.

Author Contributions: P.N.P. and A.V.K. conceived the research. J.C.B., A.A.P., G.T., S.M.K., and A.K. designed the experiments. J.C.B. and A.A.P. fabricated and characterized bismuth nanomaterials. J.C.B., A.A.P., G.T., S.M.K., P.N.P, A.K., and A.V.K. analyzed and discussed the obtained results. J.C.B., A.A.P., P.N.P., and A.V.K. contributed to writing the manuscript with comments from all authors. P.N.P. and A.V.K. guided the project. All authors have read and agreed to the published version of the manuscript.

Funding: The research at the Institute for Lasers, Photonics and Biophotonics at Buffalo was supported by funds from the Office of Vice President for Research and Economic Development at the University at Buffalo. The authors acknowledge contribution of Russian Science Foundation (Project 19-72-30012) for the fabrication of highly calibrated NPs. Part of this work was completed using the resources of the Chemistry Instrument Center (CIC), University at Buffalo, SUNY, Buffalo, NY, mail to che-ic@buffalo.edu for sample inquiries.

Conflicts of Interest: The authors declare no conflicts of interest. 


\section{References}

1. Lee, N.; Choi, S.H.; Hyeon, T. Nano-Sized CT Contrast Agents. Adv. Mater. 2013, 25, 2641-2660. [CrossRef] [PubMed]

2. Bi, H.; He, F.; Dong, Y.; Yang, D.; Dai, Y.; Xu, L.; Lv, R.; Gai, S.; Yang, P.; Lin, J. Bismuth Nanoparticles with "Light" Property Served as a Multifunctional Probe for X-ray Computed Tomography and Fluorescence Imaging. Chem. Mater. 2018, 30, 3301-3307. [CrossRef]

3. Yu, X.; Li, A.; Zhao, C.; Yang, K.; Chen, X.; Li, W. Ultrasmall Semimetal Nanoparticles of Bismuth for Dual-Modal Computed Tomography/Photoacoustic Imaging and Synergistic Thermoradiotherapy. ACS Nano 2017, 11, 3990-4001. [CrossRef] [PubMed]

4. Song, G.; Cheng, L.; Chao, Y.; Yang, K.; Liu, Z. Emerging Nanotechnology and Advanced Materials for Cancer Radiation Therapy. Adv. Mater. 2017, 29. [CrossRef]

5. De La Vega, J.C.; Häfeli, U.O. Utilization of nanoparticles as X-ray contrast agents for diagnostic imaging applications. Contrast Media Mol. Imaging 2015, 10, 81-95. [CrossRef]

6. Deng, J.; Xu, S.; Hu, W.; Xun, X.; Zheng, L.; Su, M. Tumor targeted, stealthy and degradable bismuth nanoparticles for enhanced X-ray radiation therapy of breast cancer. Biomaterials 2018, 154, 24-33. [CrossRef]

7. Liu, C.; Zhang, L.; Chen, X.; Li, S.; Han, Q.; Li, L.; Wang, C. Biomolecules-assisted synthesis of degradable bismuth nanoparticles for dual-modal imaging-guided chemo-photothermal therapy. Chem. Eng. J. 2020, 382, 122720. [CrossRef]

8. Bierer, D.W. Bismuth Subsalicylate: History, Chemistry, and Safety. Rev. Infect. Dis. 1990, 12, S3-S8. [CrossRef]

9. Wang, F.; Tang, R.; Yu, H.; Gibbons, P.C.; Buhro, W.E. Size- and Shape-Controlled Synthesis of Bismuth Nanoparticles. Chem. Mater. 2008, 20, 3656-3662. [CrossRef]

10. Carotenuto, G.; Hison, C.L.; Capezzuto, F.; Palomba, M.; Perlo, P.; Conte, P. Synthesis and thermoelectric characterisation of bismuth nanoparticles. J. Nanoparticle Res. 2008, 11, 1729. [CrossRef]

11. Wei, B.; Zhang, X.; Zhang, C.; Jiang, Y.; Fu, Y.-Y.; Yu, C.; Sun, S.-K.; Yan, X.-P. Facile Synthesis of Uniform-Sized Bismuth Nanoparticles for CT Visualization of Gastrointestinal Tract in Vivo. ACS Appl. Mater. Interfaces 2016, 8, 12720-12726. [CrossRef] [PubMed]

12. Kabashin, A.V.; Delaporte, P.; Pereira, A.; Grojo, D.; Torres, R.; Sarnet, T.; Sentis, M. Nanofabrication with Pulsed Lasers. Nanoscale Res. Lett. 2010, 5, 454. [CrossRef]

13. Zhang, D.; Gökce, B.; Barcikowski, S. Laser Synthesis and Processing of Colloids: Fundamentals and Applications. Chem. Rev. 2017, 117, 3990-4103. [CrossRef] [PubMed]

14. Fojtik, A.; Henglein, A. Laser ablation of films and suspended particles in a solvent: Formation of cluster and colloid solutions. Ber. Bunsen-Ges. 1993, 97, 252-254.

15. Mafuné, F.; Kohno, J.-Y.; Takeda, Y.; Kondow, T.; Sawabe, H. Formation of Gold Nanoparticles by Laser Ablation in Aqueous Solution of Surfactant. J. Phys. Chem. B 2001, 105, 5114-5120. [CrossRef]

16. Kabashin, A.V.; Meunier, M. Synthesis of colloidal nanoparticles during femtosecond laser ablation of gold in water. J. Appl. Phys. 2003, 94, 7941-7943. [CrossRef]

17. Hebié, S.; Holade, Y.; Maximova, K.; Sentis, M.; Delaporte, P.; Kokoh, K.B.; Napporn, T.W.; Kabashin, A.V. Advanced Electrocatalysts on the Basis of Bare Au Nanomaterials for Biofuel Cell Applications. ACS Catal. 2015, 5, 6489-6496. [CrossRef]

18. Bailly, A.-L.; Correard, F.; Popov, A.; Tselikov, G.; Chaspoul, F.; Appay, R.; Al-Kattan, A.; Kabashin, A.V.; Braguer, D.; Esteve, M.-A. In vivo evaluation of safety, biodistribution and pharmacokinetics of laser-synthesized gold nanoparticles. Sci. Rep. 2019, 9, 12890. [CrossRef]

19. Baati, T.; Al-Kattan, A.; Esteve, M.-A.; Njim, L.; Ryabchikov, Y.; Chaspoul, F.; Hammami, M.; Sentis, M.; Kabashin, A.V.; Braguer, D. Ultrapure laser-synthesized Si-based nanomaterials for biomedical applications: In vivo assessment of safety and biodistribution. Sci. Rep. 2016, 6, 25400. [CrossRef]

20. Kabashin, A.V.; Singh, A.; Swihart, M.T.; Zavestovskaya, I.N.; Prasad, P.N. Laser-Processed Nanosilicon: A Multifunctional Nanomaterial for Energy and Healthcare. ACS Nano 2019, 13, 9841-9867. [CrossRef]

21. Popov, A.A.; Tselikov, G.; Dumas, N.; Berard, C.; Metwally, K.; Jones, N.; Al-Kattan, A.; Larrat, B.; Braguer, D.; Mensah, S.; et al. Laser- synthesized TiN nanoparticles as promising plasmonic alternative for biomedical applications. Sci. Rep. 2019, 9, 1194. [CrossRef] [PubMed] 
22. Popova-Kuznetsova, E.; Tikhonowski, G.; Popov, A.A.; Duflot, V.; Deyev, S.; Klimentov, S.; Zavestovskaya, I.; Prasad, P.N.; Kabashin, A.V. Laser-Ablative Synthesis of Isotope-Enriched Samarium Oxide Nanoparticles for Nuclear Nanomedicine. Nanomaterials 2019, 10, 69. [CrossRef] [PubMed]

23. Gondal, M.A.; Saleh, T.A.; Drmosh, Q. Optical Properties of Bismuth Oxide Nanoparticles Synthesized by Pulsed Laser Ablation in Liquids. Sci. Adv. Mater. 2012, 4, 507-510. [CrossRef]

24. Escobar-Alarcón, L.; Velarde Granados, E.; Solís-Casados, D.A.; Olea-Mejía, O.; Espinosa-Pesqueira, M.; Haro-Poniatowski, E. Preparation of bismuth-based nanosheets by ultrasound-assisted liquid laser ablation. Appl. Phys. A 2016, 122, 433. [CrossRef]

25. Dadashi, S.; Poursalehi, R.; Delavari H, H. Formation, gradual oxidation mechanism and tunable optical properties of $\mathrm{Bi} / \mathrm{Bi}_{2} \mathrm{O}_{3}$ nanoparticles prepared by Nd:YAG laser ablation in liquid: Dissolved oxygen as genesis of tractable oxidation. Mater. Res. Bull. 2018, 97, 421-427. [CrossRef]

26. Dadashi, S.; Poursalehi, R.; Delavari, H. Optical and structural properties of Bi-based nanoparticles prepared via pulsed Nd:YAG laser ablation in organic liquids. Appl. Phys. A 2018, 124, 406. [CrossRef]

27. Dadashi, S.; Delavari, H.; Poursalehi, R. Optical Properties and Colloidal Stability Mechanism of Bismuth Nanoparticles Prepared by Q-switched Nd:Yag Laser Ablation in Liquid. Procedia Mater. Sci. 2015, 11,679-683. [CrossRef]

28. Kumar, A.; Dixit, C.K. 3-Methods for characterization of nanoparticles. In Advances in Nanomedicine for the Delivery of Therapeutic Nucleic Acids; Nimesh, S., Chandra, R., Gupta, N., Eds.; Woodhead Publishing: Cambridge, UK, 2017; pp. 43-58. [CrossRef]

29. Huang, H.; Tian, N.; Jin, S.; Zhang, Y.; Wang, S. Syntheses, characterization and nonlinear optical properties of a bismuth subcarbonate $\mathrm{Bi}_{2} \mathrm{O}_{2} \mathrm{CO}_{3}$. Solid State Sci. 2014, 30, 1-5. [CrossRef]

30. Dong, F.; Zheng, A.; Sun, Y.; Fu, M.; Jiang, B.; Ho, W.-K.; Lee, S.C.; Wu, Z. One-pot template-free synthesis, growth mechanism and enhanced photocatalytic activity of monodisperse $(\mathrm{BiO})_{2} \mathrm{CO}_{3}$ hierarchical hollow microspheres self-assembled with single-crystalline nanosheets. CrystEngComm 2012, 14, 3534-3544. [CrossRef]

31. Marinho, J.Z.; Santos, L.M.; Macario, L.R.; Longo, E.; Machado, A.E.H.; Patrocinio, A.O.T.; Lima, R.C. Rapid Preparation of $(\mathrm{BiO})_{2} \mathrm{CO}_{3}$ Nanosheets by Microwave-Assisted Hydrothermal Method with Promising Photocatalytic Activity Under UV-Vis Light. J. Braz. Chem. Soc. 2015, 26, 498-505.

32. Xiong, T.; Huang, H.; Sun, Y.; Dong, F. In situ synthesis of a C-doped $(\mathrm{BiO}){ }_{2} \mathrm{CO}_{3}$ hierarchical self-assembly effectively promoting visible light photocatalysis. J. Mater. Chem. A 2015, 3, 6118-6127. [CrossRef]

33. Hardcastle, F.D.; Wachs, I.E. The molecular structure of bismuth oxide by Raman spectroscopy. J. Solid State Chem. 1992, 97, 319-331. [CrossRef]

34. Lewis, J.S.a.R. In situ micro-Raman studies of laser-induced bismuth oxidation reveals metastability of $\beta-\mathrm{Bi}_{2} \mathrm{O}_{3}$ microislands. Opt. Mater. Express 2014, 4, 2133-2144.

35. Taylor, P.; Sunder, S.; Lopata, V.J. Structure, spectra and stability of solid bismuth carbonates. Can. J. Chem. 1984, 62, 2863-2873. [CrossRef]

36. Wang, Y.W.; Hong, B.H.; Kim, K.S. Size Control of Semimetal Bismuth Nanoparticles and the UV-Visible and IR Absorption Spectra. J. Phys. Chem. B 2005, 109, 7067-7072. [CrossRef]

37. Wang, Z.; Jiang, C.; Huang, R.; Peng, H.; Tang, X. Investigation of Optical and Photocatalytic Properties of Bismuth Nanospheres Prepared by a Facile Thermolysis Method. J. Phys. Chem. C 2014, 118, 1155-1160. [CrossRef]

38. Huang, Y.; Wang, W.; Zhang, Q.; Cao, J.-j.; Huang, R.-j.; Ho, W.; Lee, S.C. In situ Fabrication of $\alpha-\mathrm{Bi}_{2} \mathrm{O}_{3} /(\mathrm{BiO})_{2} \mathrm{CO}_{3}$ Nanoplate Heterojunctions with Tunable Optical Property and Photocatalytic Activity. Sci. Rep. 2016, 6, 23435. [CrossRef]

39. Dong, F.; Li, Q.; Sun, Y.; Ho, W.-K. Noble Metal-Like Behavior of Plasmonic Bi Particles as a Cocatalyst Deposited on $(\mathrm{BiO})_{2} \mathrm{CO}_{3}$ Microspheres for Efficient Visible Light Photocatalysis. ACS Catal. 2014, 4, 4341-4350. [CrossRef]

(C) 2020 by the authors. Licensee MDPI, Basel, Switzerland. This article is an open access article distributed under the terms and conditions of the Creative Commons Attribution (CC BY) license (http://creativecommons.org/licenses/by/4.0/). 